\title{
Hitler, Mein Kampf. Eine kritische Edition
}

\section{François Delpla}

\section{(2) OpenEdition \\ Journals}

Édition électronique

URL : http://journals.openedition.org/ifha/8992

DOI : 10.4000/ifha.8992

ISSN : 2198-8943

Éditeur

IFRA - Institut franco-allemand (sciences historiques et sociales)

\section{Référence électronique}

François Delpla, « Hitler, Mein Kampf. Eine kritische Edition », Revue de l'IFHA [En ligne], Date de recension, mis en ligne le 10 janvier 2018, consulté le 23 septembre 2020. URL : http:// journals.openedition.org/ifha/8992 ; DOI : https://doi.org/10.4000/ifha.8992

Ce document a été généré automatiquement le 23 septembre 2020.

(CIFHA 


\section{Hitler, Mein Kampf. Eine kritische Edition}

\section{François Delpla}

\section{RÉFÉRENCE}

Hitler, Mein Kampf. Eine kritische Edition, herausgegeben im Auftrag des Instituts für Zeitgeschichte München - Berlin von Christian Hartmann, Thomas Vordermayer, Othmar Plöckinger, Roman Töppel, München: Institut für Zeitgeschichte, 2016, 1948 p., $59 €$ 
Othmar Plöckinger, Christian Hartmann, Thomas Vordermayer et Roman Töppel ont fabriqué un objet lourd et beau, décourageant l'avarice, la paresse et la précipitation. Il faut, même si l'on connaissait préalablement l'œuvre d'Hitler, conquérir pas à pas l'introduction générale, l'introduction de chaque chapitre et, surtout, les notes. Du moins, en contemplant l'objet de l'extérieur, ou en se remémorant les soubresauts qui ont accompagné sa gestation, comprend-on d'un coup, sans même avoir besoin de l'ouvrir, que l'unique livre publié par Adolf Hitler est autre chose qu'un «fatras de lectures mal digérées » (suivant l'expression consacrée par des décennies d'antinazisme myope) et qu'il est, au contraire, original et important.

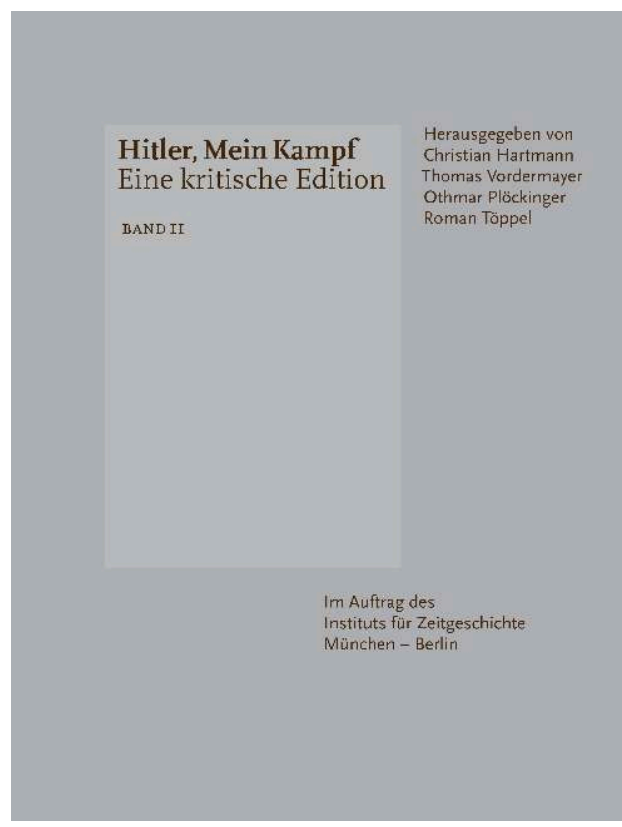

Les auteurs le dédoublent: Mein Kampf est à la fois un agent historique et un « symbole encore actif ». Cette édition vise à désactiver le symbole par le dévoilement de ses effets criminels. Car la mise en évidence du caractère d'agent historique de l'ouvrage autrement dit de sa place dans l'histoire de l'Allemagne et celle du nazisme contribuera puissamment, espèrent les auteurs, à le démonétiser en tant que manifeste politique.

Une information qui surprendra beaucoup de lecteurs est le caractère solitaire de l'écriture de ce livre et le peu d'influence de l'entourage de Hitler tant sur la forme que sur le fond, fût-ce au moment de la relecture. L'auteur est unique et, s'il n'invente pas tout, du moins fait-il de ses lectures un usage personnel, aux antipodes du préjugé selon lequel il aurait recopié bout à bout des développements sans bien les comprendre. Cette découverte, bien qu'elle ait déjà figuré dans les ouvrages de l'un des quatre éditeurs, Othmar Plöckinger, depuis une décennie, tarde à irriguer les analyses historiques et il faut espérer que cette parution fera sauter un verrou à cet égard.

Un autre jugement classique porte sur la langue, un très mauvais allemand alternant platitudes et boursouflures, faisant de la lecture un chemin de croix. En fait, Hitler écrit " comme beaucoup d'auteurs de son temps » et trouve par moments des expressions heureuses ou des images frappantes. Les auteurs n'hésitent pas à citer favorablement (p. 24) un article de 2014 dont le titre, " Hitler était-il un bon écrivain ? ", aurait sonné, il y a quelques années encore, comme une provocation: "Dans les oreilles d'"adeptes réels ou potentiels" ou de lecteurs innocents ou "non informés", qui n'abordaient pas Mein Kampf en prétendant regarder de façon critique la présentation de Hitler par luimême, le livre pouvait très bien, avec son style qui nous est aujourd'hui étranger, "susciter aussi de l'admiration et créer des partisans". Il ne faut jamais, au cours de la lecture, perdre cela de vue. »

Le livre est présenté, un peu excessivement peut-être, comme un commencement. En particulier, pour Hitler, il s'agirait de la première rencontre avec la réalité d'une 
existence jusque-là gangrenée d'irréalisme. Le fait de pouvoir se dire « écrivain » et de se faire rémunérer pour un travail représenterait un saut qualitatif : «Pour la première fois Hitler avait obtenu avec Mein Kampf quelque chose qui correspondait à la conception bourgeoise d'une existence assurée, également parce qu'il gagnait pour cela de l'argent liquide» (p.29). Et de citer, au mark près, le gain du premier trimestre, comme si le parvenu Hitler l'avait touché en espèces et l'avait recompté avec jubilation.

Le jugement de valeur fait parfois de l'ombre à la description : « La référence constante du livre à l'histoire de sa vie personnelle en dit long sur la partialité égocentrique de l'auteur» (p. 26). Non seulement ce moralisme n'ajoute rien à l'analyse, mais il peut induire en erreur. Ce serait en vertu de son égocentrisme que Hitler laisserait dans l'ombre tous les membres de sa famille à part son père et sa mère puis, racontant sa guerre, qu'il ne nommerait pas ses compagnons. Pour la même raison, il passerait sous silence les talents militaires déployés pendant la guerre par son acolyte du putsch manqué, le général Ludendorff (p. 41). Enfin, dans les pages sur l'histoire du parti nazi, c'est encore par égocentrisme qu'il rapporterait tout à sa personne. Faudrait-il rappeler qu'il s'agit d'un manifeste politique, et que la politique qu'il propose consiste précisément à héroïser un chef et un seul ? On pourrait aussi bien, et sans doute mieux, remarquer que peu d'écrits politiques donnent autant de détails sur la vie familiale et, quand ils n'ont rien de saillant, les états de service militaires de leur auteur. Le livre remarque tout de même, fort justement, que la croix-de-fer de première classe n'était pas souvent donnée à un soldat qui ne fût pas officier; mais ce n'est tout de même pas une décoration rare et tout autre serait ridicule de s'en vanter : si Hitler le fait, ce n'est pas pour se distinguer de la foule mais, précisément, pour souligner sa condition de héros surgi du peuple.

Le reproche (p. 35-36) concernant l'histoire du Parti (il n'y en a que pour Hitler, très peu pour ses camarades) pourrait être tempéré au moyen d'un ouvrage complémentaire, souvent cité ici à d'autres propos: les «Monologues au quartiergénéral » (d'après le titre allemand, assez infidèle au contenu), parus en 1952-53 dans une version française de mauvaise qualité et récemment édités en France dans une traduction enfin convenable sous le titre Propos intimes et politiques. Dans ces conversations de 1941-42, recueillies et mises au net sous la direction de Martin Bormann, Hitler revient longuement (probablement dans le dessein de compléter Mein Kampf au cas où la guerre lui en ôterait le loisir) sur les premières années du NSDAP, en nommant beaucoup de ses membres, de façon bienveillante en général. Reste à savoir si ces considérations auraient été à leur place dans un livre-manifeste, destiné à poser les bases essentielles d'une doctrine et d'un programme.

On pourrait même se demander si des traits présentés de façon péjorative ne seraient pas, chez tout autre, salués comme de précieuses qualités. Ainsi p. 42, lorsqu'est stigmatisé le fait qu'Hitler évoque les militants des partis concurrents de la mouvance völkisch "sans citer aucun nom»: damnatio memoriae, vraiment? Ou parti pris intelligent d'aller de l'avant en oubliant les querelles de personnes et en facilitant les regroupements? D'ailleurs, si l'on tient à dire systématiquement du mal de l'œuvre, ce serait le moment d'en évoquer le nationalisme étroit et le racisme exacerbé : si Hitler passe l'éponge sur le passé, ce n'est pas par grandeur d'âme mais bien parce qu'il conçoit le peuple allemand comme un tout organique dont le "sang", en chaque élément, est excellent. Les fautes doivent donc faire l'objet d'une large miséricorde, pour peu que le sang soit pur, le repentir sincère, et sans arrière-pensées le ralliement 
au Führer nazi. Ce serait aussi l'occasion d'une comparaison pertinente avec le stalinisme, adepte, lui, de la damnatio memoriae et des rancunes indélébiles... et, de ce fait, beaucoup moins attractif. Enfin on pourrait rappeler que l'égocentrisme n'exclut pas complètement le souvenir ni la vengeance, témoin la liquidation lors de «la nuit des longs couteaux » de quelques anciens adversaires au sein de la réaction bavaroise, comme Kahr, Ballerstedt et Gerlich, ou encore l'incarcération fatale à la santé de l'ancien ministre de l'Intérieur Franz Xaver Schweyer, un conservateur obstinément antinazi, décédé en 1935 peu après sa libération.

Une surprise attend le lecteur non prévenu (p. 38) : l'absence du mot "dictature ", sinon en un sens péjoratif, pour dénigrer des adversaires. Hitler préfère... la démocratie, à condition, il est vrai, qu'elle soit flanquée de l'adjectif "allemande ". Cependant, le lecteur de l'introduction, à peine remis de cette surprise, est renvoyé vers une note du chapitre 12 (le dernier du premier tome) qui précise que la démocratie allemande a disparu dans l'édition de 1930 - un épisode particulièrement symptomatique et jusqu'ici peu remarqué. Le texte original « Le mouvement pose, sur les plus grandes comme sur les plus petites questions, le principe d'une démocratie allemande : élection du Führer, mais reconnaissance de son autorité absolue » devient en 1930, trois ans avant la prise du pouvoir : «Le mouvement pose, sur les plus grandes comme sur les plus petites questions, le principe de l'autorité absolue du Führer, couplée avec la responsabilité la plus totale.» Toutefois, la démocratie allemande et l'élection du Führer survivent jusqu'en 1945 au chapitre 3 (p. 90 et 94). Voilà qui demanderait de nouvelles recherches pour savoir si la correction a été mise en avant et si l'ancienne version a subsisté par erreur, ou si elle est restée, dans les citations, les articles, etc., aussi fréquente que l'autre, peut-être à l'usage de publics différents ou en fonction des besoins politiques du moment.

La question du caractère prémonitoire de Mein Kampf est abordée de front mais résolue de manière inégale. Autant le tropisme guerrier du nazisme est cerné avec justesse, autant l'esquisse des futures opérations est dénigrée avec naïveté. Hitler est censé (p. 47) s'être mépris sur la disposition du Royaume-Uni à s'allier avec lui. Londres aurait pu consentir, concèdent les auteurs, à s'entendre avec une Allemagne "modérée ", mais certainement pas avec un tel trublion. C'est là sous-estimer à la fois les compromissions des appeasers entre 1933 et 1939 et les efforts aux résultats longtemps incertains de Churchill, ensuite, pour renverser la tendance et persuader le monde qu'il avait réellement converti les élites britanniques à l'antinazisme. Cette sous-estimation va de pair avec celle du danger couru par la France. Certes le projet d'une guerre contre elle est mentionné (p.49), mais pour s'en gausser. Au lieu de saluer une anticipation exacte ou, mieux, un projet audacieux réalisé grâce à une débauche de ténacité, de dissimulation et d'intelligence, voilà qu'on s'en tient à la date de publication du deuxième tome, 1926, pour railler le donquichottisme de l'habitant d'un pays à terre qui prétend terrasser une puissance à son zénith.

Quant au caractère prémonitoire ou non des considérations sur les Juifs, nos germaniques auteurs se font, dirait-on en France, normands : «Dire que les éructations de haine de Mein Kampf tracent vers Auschwitz un chemin rectiligne serait beaucoup trop simple. Inversement, dire qu'il n'y a aucun rapport serait tout aussi erroné. » Il conviendrait de remarquer qu'à elles seules les métaphores médicales ou animales qui caractérisent le peuple désigné par Hitler comme l'ennemi du genre humain (bacilles, pestilence, abcès, pourriture, parasite, poison, cancer, gangrène, rats, araignée, etc.) 
appellent une destruction violente et complète. Elle est, de surcroit, urgente puisque la lutte de cette entité avec la prétendue race aryenne est censée avoir atteint un stade terminal. Cela est dit, d'ailleurs : pourquoi ne pas en déduire que la route vers quelque Auschwitz est plus nettement dessinée que la direction inverse ?

Après cette longue et riche introduction, le texte de Mein Kampf occupe environ le tiers de la page de droite, le reste et celle de gauche étant réservés aux variantes des éditions successives, et surtout aux notes, dont la vocation essentielle est d'empêcher le lecteur de croire sans examen ce qu'il lit. Parfois ces notes débordent sur la double page suivante et le texte d'Hitler ne reprend que deux pages plus loin. Il peut aussi arriver qu'elles soient peu nombreuses et que de vastes blancs subsistent. Ainsi, p. 527, une page dénonçant les automutilations des soldats "lâches" n'appelle pas d'autre commentaire qu'une courte évocation du phénomène au sein de l'armée allemande en général et du régiment List en particulier, d'après les travaux de Ziemann et de Weber. Plus fournie est la double page suivante, où les Juifs sont accusés de toutes les pratiques antipatriotiques possibles: des notes abondantes font comprendre d'une part que la dégradation de la situation militaire ou alimentaire tenait à des causes générales, d'autre part que les Juifs allemands prenaient toute leur part des sacrifices, cependant que l'affaire du "recensement juif» de 1916 fait l'objet de précisions utiles. Plus surprenant est le désert qui entoure, p. 555 et 557, le récit par Hitler de son séjour à la clinique de Pasewalk et de la crise aux allures mystiques, accompagnée d'une rechute dans la cécité, qu'il vécut d'après ses dires à l'annonce de l'armistice. Seulement deux notes, une par page, pour dire que Hitler démarque l'inscription des Thermopyles à la mémoire des soldats de Léonidas, puis pour commenter la phrase finale sur la décision d'Hitler d'entrer en politique, en remarquant qu'elle inversait la fameuse maxime de Clausewitz. La crise elle-même est laissée sans commentaire alors qu'elle fait l'objet d'une littérature importante et contradictoire depuis les travaux de Rudolph Binion dans les années 1970.

Voilà qui nous amène à la question principale, celle de la conversion d'Hitler à sa propre idéologie et, en particulier, à l'antisémitisme - avec la coloration particulière que cette très ancienne phobie a prise chez lui. Il expose longuement sa version des choses dans les dernières pages du chapitre 2 et peut-être aussi, ce qui est moins souvent remarqué, dans la première du chapitre suivant. Inaugurée en 1996 par Brigitte Hamann, la remise en cause de sa chronologie est l'objet, depuis, d'un vif débat. Sans préciser aucune date, Hitler s'efforce de faire croire qu'il est devenu antisémite peu après son arrivée à Vienne, soit vers l'âge de vingt ans. Suivant une pente qui amenait tous les auteurs ou presque, jusqu'à une époque récente, à croire les nazis sur parole dès lors qu'ils disaient du mal d'eux-mêmes, ce récit a longtemps reçu une confiance unanime et, dès lors, on ne fit aucune difficulté pour admettre le témoignage du vieil idéologue viennois Lanz, dit Lanz von Liebenfels, directeur dans les années 1900 du magazine antisémite Ostara, lorsqu'il prétendit en 1951 que le jeune Hitler lui avait un jour rendu visite pour compléter sa collection. B. Hamann, suivie de quelques auteurs sans doute encore minoritaires, a la première mis en doute ce récit dans son Hitlers Wien. Les auteurs lui emboîtent le pas sans s'étendre sur le sujet, trouvant " douteuse » l'influence de Lanz sur Hitler (p. 209, note 1). Mais ils ne se mettent pas à la recherche d'une chronologie alternative. Or les premières lignes du chapitre 3 auraient pu les y inciter. Hitler y édicte une règle curieuse : il ne faudrait pas faire de politique avant trente ans - un évident plaidoyer pro domo - et il ne faudrait pas non plus confier de fonction éminente à quelqu'un qui, après cet âge, aurait changé d'avis sur un point 
important. Max Domarus, le premier et l'un des rares à commenter ce passage (en 1964, dans l'introduction de son édition des discours et articles d'Hitler), l'attribue à un souci de d'imiter Jésus-Christ. Ici (p.234, n. 1), les auteurs considèrent qu'Hitler a pu être influencé par l'âge requis pour les fonctions publiques en Grèce et à Rome ou par les lois autrichiennes d'avant 1914. Or il s'agit peut-être d'une clé : Hitler aurait lui-même " changé d'avis sur un point important » peu avant son trentième anniversaire, échu le 20 avril 1919. La question mériterait au moins d'être posée et cela nous ramène à R. Binion, qui situe le basculement de la vie d'Hitler à Pasewalk, dans les affres de la défaite de 1918, lesquels auraient entraîné le déclenchement d'une psychose paranoïaque, jusque-là latente. Il aurait pu alors flotter quelque temps dans la définition de son ennemi, et opter pour «le Juif» lors des troubles munichois d'avril 1919.

Ces lacunes sont peut-être en rapport avec une certaine étroitesse de la bibliographie. Si certains ouvrages récents, par exemple la biographie politique d'Hitler par Wolfram Pyta, sont intelligemment exploités, la grande majorité des travaux cités sont allemands et la production étrangère est, à part quelques Britanniques incontournables, à peu près ignorée. Pour ne citer que quelques exemples en sus de celui de R. Binion, l'Américain John Lukacs, pionnier de la découverte des qualités manœuvrières d'Hitler, est passé sous silence. Quant à son compatriote Gerhard Weinberg, découvreur du Second livre et initiateur des études sur la politique extérieure du Reich, il n'est mentionné (p. 1884) que pour avoir traité d'une question ponctuelle dans un ouvrage collectif.

$\mathrm{Au}$ total, si ce livre ne marque, dans l'analyse du nazisme et la compréhension son fondateur, qu'un progrès mesuré, il ne décevra pas son possesseur en tant qu'instrument de travail propre à favoriser une accélération des recherches et des découvertes.

INDEX

Index chronologique : Époque contemporaine

Thèmes : Sources 